\title{
Influência do Fósforo e do Potássio na Severidade da Ferrugem da Soja Phakopsora pachyrhizi
}

\author{
Ricardo S. Balardin, Leandro J. Dallagnol, Henrique T. Didoné \& Lucas Navarini \\ Departamento de Defesa Fitossanitária, Centro de Ciências Rurais, Universidade Federal de Santa Maria, \\ Cx. Postal 5025, CEP 97111-970, Santa Maria, RS, e-mail: rsbalardin@balardin.com.br
}

(Aceito para publicação em 17/11/2006)

Autor para correspondência: Ricardo Silveiro Balardin

BALARDIN, R. S., DALlaGNOL, L. J., DIDONÉ, H.T. \& NAVARINI, L. Influência do Fósforo e do Potássio na Severidade da Ferrugem da Soja Phakopsora pachyrhizi. Fitopatologia Brasileira 31:462-467. 2006.

\section{RESUMO}

O fornecimento de nutrientes minerais para a cultura da soja favorece a redução da severidade de Phakopsora pachyrhizi. As cultivares de soja "Embrapa 48" e "Al 83", utilizadas no experimento, apresentam expressão diferenciada da resistência parcial, sendo a "Embrapa 48" mais resistente que a "Al 83". As cultivares foram semeadas em vasos plásticos com substrato composto de areia lavada, terra argilosa e casca de arroz não carbonizada (3:1:2). Os tratamentos adicionados ao substrato, foram constituídos de diferentes níveis de fósforo $(\mathrm{P})$, potássio $(\mathrm{K})$ e combinações. A inoculação das plantas com uredosporos de P. pachyrhizi foi realizada nos estádios fenológicos V2 e R5. Para avaliação foi utilizada a severidade da ferrugem medida aos 14, 17, 21 e 25 dias após a inoculação, sendo calculada a taxa de progresso. Os resultados mostraram influência significativa da nutrição da planta, reduzindo tanto a severidade final como a taxa de progresso da ferrugem com o aumento nos níveis de $\mathrm{P}$ e $\mathrm{K}$, para os estádios e cultivares testados. A maior redução foi verificada no estádio V2. A expressão da resistência parcial nas cultivares utilizadas foi influenciada pela variação nos níveis de P e K, sendo em baixas dosagens de P e K, observado maior diferença na expressão no período de latência do fungo. A associação de cultivares com resistência parcial elevada e nutrição mineral equilibrada, são elementos para um programa de manejo integrado que possibilitam maior eficiência no controle da ferrugem da soja, em adição às estratégias com base na evasão e proteção.

Palavras-chave adicionais: manejo de fitodoença, nutrição mineral, resistência genética.

\begin{abstract}
Influence of phosphorus and potassium on severity of soy bean rust, Phakopsora pachyrhizi

Mineral nutrition on soybean might reduce the severity of Phakopsora pachyrhizi. "Embrapa 48" showed higher level of partial resistance than "Al 83" cultivars. Both cultivars were utilized in the trials. The cultivars were planted in plastic pots with sandy soil, clay and unburned rice husks (3:1:2). The treatments added to the substract were different levels of phosphorus and potassium. The plants were inoculated with uredospores of $P$. pachyrhizi at V2 and R5 stages, and rust severity evaluated at $14,17,21$ and 25 days after inoculation. The disease progress rate was calculated. The results showed significant influence of plant nutrition, reducing both the final severity and disease progress rate as $\mathrm{P}$ and $\mathrm{K}$ level increased. The greatest reduction was observed at V2 stage. Greater disease reduction was observed at lower levels of K. The expression of partial resistance of both cultivars was influenced due to variation of $\mathrm{P}$ and $\mathrm{K}$ levels. Lower doses of $\mathrm{P}$ and $\mathrm{K}$ induced a greater difference in the latent period of the pathogen. The association of genetic cultivar background to mineral nutrition might result in an integrated management disease program, along with evasion and chemical protection strategies.
\end{abstract}

Additional keywords: Plant disease management, mineral nutrition, genetic resistance.

\section{INTRODUÇÃO}

A nutrição das plantas, considerada como um fator ambiente pode alterar a reação das plantas aos patógenos, influenciando o progresso da doença. $\mathrm{O}$ suprimento balanceado de nutrientes que favorece o crescimento normal das plantas é também considerado como relevante para seus processos de defesa (Marschner, 1986).

A deficiência, excesso ou desequilíbrio nas combinações de elementos nutricionais, pode influenciar a reação das plantas à infecção por patógeno de forma a aumentar o nível de defesa ou favorecer a ocorrência de doenças. Em videira, a redução da severidade do oídio Uncinula necator (Schwein.) Burrill ocorre com altos níveis de nitrogênio $(\mathrm{N})$, fósforo $(\mathrm{P})$ e potássio $(\mathrm{K})$ via fertirrigação (Reuveni et al. 1993). Os autores atribuíram o efeito principalmente ao $\mathrm{P}$, o qual teria incrementado o nível de resistência ao patógeno. Para Develash \& Sugha (1997), o aumento das quantidades de $\mathrm{K}$ retardou o desenvolvimento de míldio da cebola. Em soja a incidência de Phomopsis phaseoli (Desm.) Sacc. reduziu com o aumento nas doses de K na adubação (Mascarenhas et al. 1976, Ito et al. 1994). A incidência de Cercospora coffeicola Berk. \& M.A. Curtis diminuiu em plantas de café com o aumento da adubação 
de $\mathrm{K}$ e $\mathrm{Ca}$, enquanto que a severidade foi reduzida pela interação K x Ca (Garcia Junior et al., 2003). Levi (2002) verificou que a aplicação de cloreto de potássio ou nitrato de potássio, reduziu a severidade da ferrugem asiática.

Develash \& Sugha (1997) verificaram que o aumento nas quantidades de $\mathrm{N}$ foi correlacionado com a variação na severidade de míldio da cebola. Para Nava-Juares et al. (2005), aumentos na dose de nitrogênio reduziram a população de esclerócios de Sclerotium rolfsii Sacc. e o número de bulbos de cebola com sintomas da doença. Interação significativa entre fertilização nitrogenada e a aplicação de fungicidas ocorreu para o controle da mancha amarela do trigo causada por Pyrenophora tritici-repentis (Died.) Drechsler, o aumento de $\mathrm{N}$ diminuiu a eficiência dos fungicidas testados. (Annone et al., 2005).

A redução do nível de inoculo inicial por meio da utilização de cultivares com resistência parcial associada a uma nutrição mineral equilibrada pode possibilitar um controle mais eficiente das doenças. Dallagnol (2004) verificou diferenças expressivas na resistência parcial a ferrugem da soja, nas cultivares "Embrapa 48" e "AL 83", sendo a "Embrapa 48" com período de latência do patógeno mais longo que a "AL 83".

A redução do inóculo e da taxa de progresso de doenças pode ser alcançado através da disponibilização de nutrientes ou pelo fornecimento direto dos nutrientes, ou por práticas culturais que melhorem a absorção ou a interação com o ambiente (Pozza \& Pozza 2003). Segundo Huber (2002), a integração do efeito de um nutriente específico com resistência genética, práticas culturais e controle químico podem reduzir a intensidade de doenças.

A ferrugem asiática da soja, causada pelo fungo Phakopsora pachyrhizi Syd. \& P. Syd., destacou-se pela virulência e pela elevada taxa de progresso, causando reduções significativas no rendimento de grãos da cultura. Dessa forma o objetivo do trabalho foi verificar a interação do fósforo com o potássio, em diferentes doses, na intensidade de ferrugem asiática da soja.

\section{MATERIAL E MÉTODOS}

O trabalho foi desenvolvido em casa-de-vegetação da Universidade Federal de Santa Maria, Santa Maria RS (UFSM), durante o ano de 2005. Foram realizados três experimentos, sendo o experimento 01 composto por 4 doses de potássio $\left(0,35,70\right.$ e $\left.140 \mathrm{~kg} \mathrm{ha}^{-1}\right)$ mantendo a dose de fósforo em $8,4 \mathrm{mg} \mathrm{dm}^{-3}$, O experimento 02 com 4 doses de fósforo $\left(0,42,5,85\right.$ e $\left.170 \mathrm{~kg} \mathrm{ha}^{-1}\right)$ mantendo a dose de potássio em $65 \mathrm{mg} \mathrm{dm}^{-3}$, e o terceiro experimento foram combinadas as 4 doses de potássio e as 4 doses de fósforo $(0$ e 0,35 e $42,5,70$ e 85,140 e $\left.170 \mathrm{~kg} \mathrm{ha}^{-1}\right)$.

A adubação fosfatada e potássica seguiram as recomendações apresentadas na Comissão de Química e Fertilidade do Solo (2004), visando produção superior a duas t.ha ${ }^{-1}$. As quantidades necessárias de potássio e de fósforo adicionadas ao solo foram de 70,0 e 85,0 kg.ha-1, respectivamente. As fontes utilizadas foram o superfosfato triplo (37\% de fósforo) e o cloreto de potássio $(58 \%$ de potássio). O cálculo da quantidade de superfosfato triplo e de cloreto de potássio por tratamento foi realizado por meio da formula:

$$
\begin{aligned}
& F=\left\{\left[(N \times M s \times 1000) / M S h a^{-1}\right] \times C\right\}, \text { onde; } \\
& F=\text { quantidade do fertilizante para a massa de } \\
& \text { substrato }(\mathrm{g}) ; \\
& N=\text { quantidade do elemento }(\mathrm{Kg}) ; \\
& M S=\text { quantidade de substrato (areia lavada+terra } \\
& \text { argilosa) que será fertilizado }(\mathrm{Kg}) \\
& M S h a^{-1}=\text { quantidade média de solo explorado pelo }
\end{aligned}
$$
sistema radicular da cultura em um hectare $(\mathrm{Kg})$. Neste caso foi utilizado $2.000 .000 \mathrm{~kg}$ de solo;

$C=$ concentração do elemento no fertilizante utilizado.

O substrato foi composto pela mistura de areia lavada (partículas $\leq 2 \mathrm{~mm}$ ) com solo argiloso (3:1), que foi submetida à análise química no Laboratório de Análises de solo da UFSM, tendo apresentado as seguintes características: textura 4, argila $170 \mathrm{~g} \mathrm{~kg}^{-1}, \mathrm{pH}$ (água 1:1) 6,0, fósforo $8,4 \mathrm{mg}$ $\mathrm{dm}^{-3}$, potássio $65,0 \mathrm{mg} \mathrm{dm}^{-3}$, enxofre $20,3 \mathrm{mg} \mathrm{dm}^{-3}$, cobre $13,0 \mathrm{mg} \mathrm{dm}^{-3}$, zinco $1,2 \mathrm{mg} \mathrm{dm}^{-3}$, boro $0,6 \mathrm{mg} \mathrm{dm}^{-3}$, cálcio 2,3 $\mathrm{cmol}_{\mathrm{c}} \mathrm{dm}^{-3}$, magnésio $0,6 \mathrm{cmol}_{\mathrm{c}} \mathrm{dm}^{-3}$ e alumínio 0,0 $\mathrm{cmol}_{\mathrm{c}} \mathrm{dm}^{-3}$.

A quantidade de substrato utilizada por tratamento foi de aproximadamente $96,0 \mathrm{~kg}$, distribuído em 16 vasos plásticos com volume de 5 litros. A mistura do fertilizante com o substrato foi realizada em misturador motorizado (1HP). A seqüência de adição no misturador foi o substrato, o fertilizante e por último a casca de arroz não carbonizada, na proporção de 2:1 com o substrato. Sementes das cultivares "Embrapa 48" e "AL 83" foram semeadas nos vasos plásticos, sendo após a emergência, desbastado para uma plântula por vaso.

Todo o trabalho foi conduzido em casa-de-vegetação, provida de um sistema de refrigeração, nebulização e aquecimento com controle parcial da temperatura do ar e umidade relativa. As paredes e o teto eram do tipo plástico duplo com injeção forçada de ar. $\mathrm{O}$ fornecimento de água foi realizado através de gotejadores tipo espaguete.

As inoculações das plantas com uredosporos de Phakopsora pachyrhizi foram realizadas na primeira folha trifoliada (V2) e no enchimento de grão (R5). As inoculações foram realizadas no período noturno, precedidas de um período de molhamento das plantas de 12 horas. A coleta dos uredosporos foi realizada a seco através de um succionador a vácuo. A deposição dos esporos sobre a folha foi obtida com um aerógrafo propelido a $\mathrm{CO}_{2}$, com pressão de trabalho de 0,5 bar. A pulverização foi realizada em ambas as faces da folha até completo molhamento foliar, sem que houvesse escorrimento. A suspensão de esporos foi composta por água, espalhante adesivo (Tween 80 a $0,1 \mu \mathrm{g} \mathrm{ml}^{-1}$ ) e uredosporos de P. pachyrhizi na concentração de $2 \times 10^{5}$ esporos.ml ${ }^{-1}$. Após a inoculação, as plantas foram mantidas sob condições de umidade relativa superior a $80 \%$, com um programa de 
nebulização $\left(15 \mathrm{~min}\right.$ dia $\left.^{-1}\right)$ e temperatura entre 15 e $30^{\circ} \mathrm{C}$.

A severidade da doença foi determinada através de notas visuais considerando a porcentagem de área foliar com sintomas visíveis da doença, calculando-se a taxa de progresso com base nas avaliações realizadas aos 14, 17, 21 e 25 dias após a inoculação. No estádio V2, as avaliações foram realizadas na primeira folha trifoliada, enquanto que no estádio R5, foram realizadas em quatro trifólios aleatórios na porção média superior da planta.

A taxa de progresso representa a quantidade média diária de aumento na severidade da doença, sendo determinada por meio da formula: $r=(1 / t) \ln \left(X / x_{d}\right)($ Campbell, 1990):

$r=$ taxa de progresso da doença;

$t=$ tempo decorrente entre a primeira e a ultima avaliação (dias);

$l n=$ logaritmo natural;

$X=$ severidade da doença na ultima avaliação (\%);

$x_{o}=$ severidade da doença na primeira avaliação(\%).

O delineamento experimental utilizado foi o de blocos ao acaso, com quatro repetições. Os dados obtidos foram submetidos ao teste de Tukey $(\mathrm{p} \leq 0,05)$ para comparação múltipla de médias.

\section{RESULTADOS E DISCUSSÃO}

A variação na quantidade adicionada ao solo de $\mathrm{P} e$ $\mathrm{K}$, bem como as combinações de dosagens, influenciaram significativamente a severidade e a taxa de progresso da ferrugem da soja.

No estádio fenológico $\mathrm{V} 2$, a variação de $0\left(65 \mathrm{mg} \mathrm{dm}^{-3}\right)$ a 140,0 kg.ha-1 $\left(65 \mathrm{mg} \mathrm{dm}^{-3}\right) \mathrm{K}$ conferiu uma redução na taxa e progresso da ferrugem de 0,43 para 0,27 . A variação de 0 $\left(8,4 \mathrm{mg} \mathrm{dm}^{-3}\right)$ a $170,0 \mathrm{~kg} \cdot \mathrm{ha}^{-1}\left(8,4 \mathrm{mg} \mathrm{dm}^{-3}\right)$ de P reduziu a taxa de progresso de 0,42 para 0,29 . No estádio R5, foi observado o mesmo comportamento observado no estádio V2, no entanto a amplitude da taxa de progresso da doença foi menor (Tabela 1). Dessa forma, a translocação dos nutrientes para os órgãos reprodutivos pode ter influenciado no desenvolvimento da doença. Segundo Potafos (1997), a redistribuição de $\mathrm{P}$ e $\mathrm{K}$ constitui-se na fonte primária desses nutrientes para os grãos em formação e resulta em significativa diminuição desses elementos na parte aérea da planta durante o período de enchimento das sementes.

$\mathrm{Na}$ cultivar "Embrapa 48", a tendência das variações na taxa de progresso da ferrugem relacionada com as variações nos níveis de nutrição das plantas mantiveram-se semelhantes às observadas na cultivar AL 83 (Tabela 2). No entanto, foi verificada redução média na taxa de progresso da ferrugem da soja de 0,35 na cultivar "AL 83 " para 0,28 na cultivar "Embrapa 48", essa apresentou aproximadamente $10 \%$ menos severidade da doença do que a cultivar "AL 83 ".

A influência do potássio foi mais pronunciada que a do fósforo quando observadas a severidade e a taxa de progresso da ferrugem, ambos em baixas quantidades. Esse resultado conferido pelo potássio, pode ser devido à ativação

TABELA 1 - Efeito da quantidade de $\mathrm{P}_{2} \mathrm{O}_{5}$ e $\mathrm{K}_{2} \mathrm{O}$, adicionados ao substrato isolados ou em combinações, sobre a severidade e taxa de progresso da ferrugem (r) da soja observadas na cultivar de soja AL 83

\begin{tabular}{|c|c|c|c|c|c|c|c|c|c|}
\hline \multirow{2}{*}{$\begin{array}{c}\mathrm{P}_{2} \mathrm{O}_{5} \\
(\mathrm{Kg} / \mathrm{ha})\end{array}$} & \multirow{2}{*}{$\begin{array}{c}\mathrm{K}_{2} \mathrm{O} \\
(\mathrm{Kg} / \mathrm{ha})\end{array}$} & \multicolumn{4}{|c|}{$\overline{\mathrm{V} 2^{2}}$} & \multicolumn{4}{|c|}{$\mathrm{R} 5^{3}$} \\
\hline & & Severidade (\%) & $\mathrm{p} \leq 0,05$ & $r^{4}$ & $\mathrm{p} \leq 0,05$ & Severidade (\%) & $\mathrm{p} \leq 0,05$ & $\mathrm{r}$ & $\mathrm{p} \leq 0,05$ \\
\hline $85\left(8,4 \mathrm{mg} \mathrm{dm}^{-3}\right)^{1}$ & $0\left(65 \mathrm{mg} \mathrm{dm}^{-3}\right)$ & 34,80 & $d^{5}$ & 0,43 & $\mathrm{c}$ & 24,00 & $\mathrm{~d}$ & 0,37 & $\mathrm{~d}$ \\
\hline $85\left(8,4 \mathrm{mg} \mathrm{dm}^{-3}\right)$ & $35\left(65 \mathrm{mg} \mathrm{dm}^{-3}\right)$ & 15,98 & $\mathrm{bc}$ & 0,34 & $\mathrm{~b}$ & 15,55 & $\mathrm{bc}$ & 0,29 & $\mathrm{bc}$ \\
\hline $85\left(8,4 \mathrm{mg} \mathrm{dm}^{-3}\right)$ & $70\left(65 \mathrm{mg} \mathrm{dm}^{-3}\right)$ & 15,00 & $\mathrm{~b}$ & 0,34 & $\mathrm{~b}$ & 13,75 & $\mathrm{~b}$ & 0,28 & $\mathrm{~b}$ \\
\hline $85\left(8,4 \mathrm{mg} \mathrm{dm}^{-3}\right)$ & $140\left(65 \mathrm{mg} \mathrm{dm}^{-3}\right)$ & 9,80 & $\mathrm{a}$ & 0,27 & $\mathrm{a}$ & 7,05 & a & 0,23 & a \\
\hline \multicolumn{2}{|c|}{$\mathrm{CV} \%$} & & \multicolumn{2}{|l|}{3,57} & 1,13 & & \multicolumn{2}{|l|}{6,50} & 3,02 \\
\hline $0\left(8,4 \mathrm{mg} \mathrm{dm}^{-3}\right)$ & $70\left(65 \mathrm{mg} \mathrm{dm}^{-3}\right)$ & 33,50 & $\mathrm{c}$ & 0,42 & $\mathrm{c}$ & 18,20 & $\mathrm{c}$ & 0,30 & $\mathrm{c}$ \\
\hline $42,5\left(8,4 \mathrm{mg} \mathrm{dm}^{-3}\right)$ & $70\left(65 \mathrm{mg} \mathrm{dm}^{-3}\right)$ & 34,00 & $\mathrm{c}$ & 0,43 & d & 13,73 & $\mathrm{~b}$ & 0,29 & $\mathrm{bc}$ \\
\hline $85\left(8,4 \mathrm{mg} \mathrm{dm}^{-3}\right)$ & $70\left(65 \mathrm{mg} \mathrm{dm}^{-3}\right)$ & 15,00 & $\mathrm{~b}$ & 0,34 & $\mathrm{~b}$ & 13,75 & $\mathrm{~b}$ & 0,28 & $\mathrm{~b}$ \\
\hline $170\left(8,4 \mathrm{mg} \mathrm{dm}^{-3}\right)$ & $70\left(65 \mathrm{mg} \mathrm{dm}^{-3}\right)$ & 10,20 & a & 0,29 & a & 8,28 & $\mathrm{a}$ & 0,25 & a \\
\hline \multicolumn{2}{|c|}{$\mathrm{CV} \%$} & & \multicolumn{2}{|l|}{3,65} & 1,26 & & \multicolumn{2}{|l|}{4,46} & 2,45 \\
\hline $0\left(8,4 \mathrm{mg} \mathrm{dm}^{-3}\right)$ & $0\left(65 \mathrm{mg} \mathrm{dm}^{-3}\right)$ & 34,66 & d & 0,44 & d & 25,88 & $\mathrm{~d}$ & 0,38 & $\mathrm{~d}$ \\
\hline $42,5\left(8,4 \mathrm{mg} \mathrm{dm}^{-3}\right)$ & $35\left(65 \mathrm{mg} \mathrm{dm}^{-3}\right)$ & 15,70 & $\mathrm{bc}$ & 0,34 & $\mathrm{bc}$ & 18,88 & $\mathrm{c}$ & 0,35 & $\mathrm{c}$ \\
\hline $85\left(8,4 \mathrm{mg} \mathrm{dm}^{-3}\right)$ & $70\left(65 \mathrm{mg} \mathrm{dm}^{-3}\right)$ & 15,00 & $\mathrm{~b}$ & 0,34 & $\mathrm{~b}$ & 13,75 & $\mathrm{~b}$ & 0,28 & $\mathrm{~b}$ \\
\hline $170\left(8,4 \mathrm{mg} \mathrm{dm}^{-3}\right)$ & $140\left(65 \mathrm{mg} \mathrm{dm}^{-3}\right)$ & 8,22 & $\mathrm{a}$ & 0,26 & $\mathrm{a}$ & 6,18 & $\mathrm{a}$ & 0,18 & $\mathrm{a}$ \\
\hline \multicolumn{2}{|c|}{$\mathrm{CV} \%$} & & 2,69 & & 1,11 & & 6,47 & & 3,00 \\
\hline
\end{tabular}

1-quantidade de nutriente presente no substrato; 2-primeiro trifolio expandido; 3-início do enchimento de grãos; 4-taxa de progresso da ferrugem asiática; 5-medias seguidas pela mesma letra na coluna não diferem pelo teste de Tukey $(\mathrm{p} \leq 0,05)$. 
TABELA 2 - Efeito da quantidade de $\mathrm{P}_{2} \mathrm{O}_{5}$ e $\mathrm{K}_{2} \mathrm{O}$, adicionados ao substrato isolados ou em combinações, sobre a severidade e taxa de progresso da ferrugem (r) da soja observadas na cultivar de soja Embrapa 48

\begin{tabular}{|c|c|c|c|c|c|c|c|c|c|}
\hline \multirow{2}{*}{$\begin{array}{c}\mathrm{P}_{2} \mathrm{O}_{5} \\
(\mathrm{Kg} / \mathrm{ha})\end{array}$} & \multirow{2}{*}{$\begin{array}{c}\mathrm{K}_{2} \mathrm{O} \\
(\mathrm{Kg} / \mathrm{ha})\end{array}$} & \multicolumn{4}{|c|}{$\mathrm{V} 2^{2}$} & \multicolumn{4}{|c|}{$\mathrm{R} 5^{3}$} \\
\hline & & Severidade $(\%)$ & $\mathrm{p} \leq 0,05$ & $r^{4}$ & $\mathrm{p} \leq 0,05$ & Severidade (\%) & $\mathrm{p} \leq 0,05$ & $\mathrm{r}$ & $\mathrm{p} \leq 0,05$ \\
\hline $85\left(8,4 \mathrm{mg} \mathrm{dm}^{-3}\right)^{1}$ & $0\left(65 \mathrm{mg} \mathrm{dm}^{-3}\right)$ & 15,80 & $c^{5}$ & 0,34 & $\mathrm{c}$ & 25,50 & $\mathrm{~d}$ & 0,37 & c \\
\hline $85\left(8,4 \mathrm{mg} \mathrm{dm}^{-3}\right)$ & $35\left(65 \mathrm{mg} \mathrm{dm}^{-3}\right)$ & 14,76 & $\mathrm{~b}$ & 0,32 & $\mathrm{~b}$ & 17,20 & $\mathrm{c}$ & 0,33 & $\mathrm{~b}$ \\
\hline $85\left(8,4 \mathrm{mg} \mathrm{dm}^{-3}\right)$ & $70\left(65 \mathrm{mg} \mathrm{dm}^{-3}\right)$ & 8,68 & $\mathrm{a}$ & 0,26 & $\mathrm{a}$ & 16,27 & $\mathrm{~b}$ & 0,33 & $\mathrm{~b}$ \\
\hline $85\left(8,4 \mathrm{mg} \mathrm{dm}^{-3}\right)$ & $140\left(65 \mathrm{mg} \mathrm{dm}^{-3}\right)$ & 8,10 & $\mathrm{a}$ & 0,25 & $\mathrm{a}$ & 10,33 & $\mathrm{a}$ & 0,24 & $\mathrm{a}$ \\
\hline \multicolumn{2}{|c|}{$\mathrm{CV} \%$} & & 3,90 & & 1,85 & & 2,12 & & 1,94 \\
\hline $0\left(8,4 \mathrm{mg} \mathrm{dm}^{-3}\right)$ & $70\left(65 \mathrm{mg} \mathrm{dm}^{-3}\right)$ & 9,04 & $\mathrm{~b}$ & 0,27 & $\mathrm{c}$ & 16,75 & $\mathrm{c}$ & 0,34 & $\mathrm{c}$ \\
\hline $42,5\left(8,4 \mathrm{mg} \mathrm{dm}^{-3}\right)$ & $70\left(65 \mathrm{mg} \mathrm{dm}^{-3}\right)$ & 8,86 & $\mathrm{~b}$ & 0,27 & $\mathrm{c}$ & 14,75 & $\mathrm{~b}$ & 0,33 & $\mathrm{~b}$ \\
\hline $85\left(8,4 \mathrm{mg} \mathrm{d} \mathrm{m}^{-3}\right)$ & $70\left(65 \mathrm{mg} \mathrm{dm}^{-3}\right)$ & 8,68 & $\mathrm{~b}$ & 0,26 & $\mathrm{~b}$ & 16,27 & $\mathrm{c}$ & 0,33 & $\mathrm{~b}$ \\
\hline $170\left(8,4 \mathrm{mg} \mathrm{dm}^{-3}\right)$ & $70\left(65 \mathrm{mg} \mathrm{dm}^{-3}\right)$ & 6,44 & $\mathrm{a}$ & 0,23 & $\mathrm{a}$ & 10,50 & $\mathrm{a}$ & 0,26 & $\mathrm{a}$ \\
\hline \multicolumn{2}{|c|}{$\mathrm{CV} \%$} & & 4,58 & & 2,10 & & 3,38 & & 1,41 \\
\hline $0\left(8,4 \mathrm{mg} \mathrm{dm}^{-3}\right)$ & $0\left(65 \mathrm{mg} \mathrm{dm}^{-3}\right)$ & 20,90 & $\mathrm{~d}$ & 0,38 & $d$ & 36,50 & $\mathrm{~d}$ & 0,42 & $\mathrm{~d}$ \\
\hline $42,5\left(8,4 \mathrm{mg} \mathrm{dm}^{-3}\right)$ & $35\left(65 \mathrm{mg} \mathrm{dm}^{-3}\right)$ & 15,02 & $\mathrm{c}$ & 0,34 & $\mathrm{c}$ & 16,83 & $\mathrm{bc}$ & 0,35 & $\mathrm{c}$ \\
\hline $85\left(8,4 \mathrm{mg} \mathrm{dm}^{-3}\right)$ & $70\left(65 \mathrm{mg} \mathrm{dm}^{-3}\right)$ & 8,68 & $\mathrm{~b}$ & 0,26 & $\mathrm{~b}$ & 16,27 & $\mathrm{~b}$ & 0,33 & $\mathrm{~b}$ \\
\hline $170\left(8,4 \mathrm{mg} \mathrm{dm}^{-3}\right)$ & $140\left(65 \mathrm{mg} \mathrm{dm}^{-3}\right)$ & 4,00 & $\mathrm{a}$ & 0,17 & $\mathrm{a}$ & 7,08 & $\mathrm{a}$ & 0,17 & $\mathrm{a}$ \\
\hline \multicolumn{2}{|c|}{$\mathrm{CV} \%$} & & 3,38 & & 1,79 & & 1,41 & & 1,23 \\
\hline
\end{tabular}

1-quantidade de nutriente presente no substrato; 2-primeiro trifolio expandido; 3-início do enchimento de grãos; 4-taxa de progresso da ferrugem asiática; 5-medias seguidas pela mesma letra na coluna não diferem pelo teste de Tukey $(\mathrm{p} \leq 0,05)$.

de enzimas envolvidas na respiração e fotossíntese, processos fornecedores de cadeias de carbono para a síntese de substâncias de defesa, bem como a regulação estomática influenciando no transporte de solutos via fluxo de massa (Bloom, 2004). Além disso, a maior disponibilidade do K na solução do solo faz desse mais facilmente absorvido pelas raízes em relação ao $\mathrm{P}$ que reage mais facilmente com as partículas do solo ficando menos disponível na solução.

A adubação equilibrada entre $\mathrm{P}$ e $\mathrm{K}$ produziu os melhores resultados para ambas as cultivares. Quando os nutrientes $\mathrm{P}$ e $\mathrm{K}$ foram reduzidos ou não adicionados na adubação, foi observada a maior severidade da ferrugem, em contrapartida, a menor severidade e a menor taxa de progresso foi observado na maior quantidade dos elementos adicionados ao solo (170 e $140 \mathrm{Kg} \cdot \mathrm{ha}^{-1}$ de P e $\mathrm{K}$, respectivamente). Melhores resultados foram obtidos, quando o aumento na quantidade de nutriente fornecido à planta foi na mesma proporção para ambos os nutrientes. Gupta \& Singh (1995) também verificaram menor incidência de míldio, Sclerospora graminicola (Sacc.) J. Schröt., em milheto, quando utilizaram NPK na adubação, em comparação a um nutriente aplicado isoladamente.

Verificou-se a influência da resistência parcial das cultivares sobre a severidade da doença (Tabela 03). A

TABELA 3 - Resumo da análise da variância considerando os dados obtidos de severidade da ferrugem de plantas sob diferentes quantidades de $\mathrm{P}_{2} \mathrm{O}_{5}$ e $\mathrm{K}_{2} \mathrm{O}$ adicionados ao substrato.

\begin{tabular}{|c|c|c|c|c|c|}
\hline Estádio Fenológico & Causas da variação & Graus de Liberdade & Soma de Quadrado & Quadrado Médio & $\mathrm{F}$ \\
\hline \multirow{7}{*}{$\mathrm{V} 2^{1}$} & Repetições & 4 & 0,69 & 0,17 & 0,47 \\
\hline & Cultivar & 1 & $2.513,02$ & 2513,02 & 6891,55 \\
\hline & Tratamentos & 9 & $5.068,26$ & 263,14 & 1544,32 \\
\hline & Cultivar $\mathrm{x}$ Tratamento & 9 & $2.130,43$ & 236,71 & 649,15 \\
\hline & Erro & 76 & 27,71 & 0,37 & \\
\hline & Total & 99 & $9.740,11$ & & \\
\hline & $\mathrm{CV} \%$ & 3,73 & & & \\
\hline \multirow{7}{*}{$\mathrm{R} 5^{2}$} & Repetições & 3 & 1,10 & 0,34 & 0,59 \\
\hline & Cultivar & 1 & 81,77 & 81,78 & 143,58 \\
\hline & Tratamentos & 9 & $4.015,08$ & 446,12 & 783,36 \\
\hline & Cultivar x Tratamento & 9 & 214,34 & 23,82 & 41,82 \\
\hline & Erro & 57 & 32,46 & 0,57 & \\
\hline & Total & 79 & $4.344,67$ & & \\
\hline & $\mathrm{CV} \%$ & 4,67 & & & \\
\hline
\end{tabular}

1-primeiro trifolio expandido; 2-início do enchimento de grãos 
interferência da cultivar sobre a severidade da doença é mais evidente na fase vegetativa, sendo reduzida durante a fase reprodutiva. Hartman et al., (1991) observaram maior sensibilidade da cultura a ferrugem da soja durante os estádios reprodutivos. A expressão da resistência parcial foi influenciada pela nutrição da planta, sendo mais pronunciada em baixas quantidades dos nutrientes adicionados ao solo (Figuras 1 - 2).

Quando foi analisada a interação da cultivar com os níveis de adubação, foi observado efeito sinergístico, independente do estádio fenológico da cultura. Dessa forma a associação de cultivares com resistência parcial elevada e nutrição mineral equilibrada pode contribuir para o estabelecimento de um programa de manejo integrado que possibilite maior eficiência no controle da ferrugem da soja, em adição a estratégia com base na evasão e proteção, no sentido de reduzir o dano da ferrugem da soja.

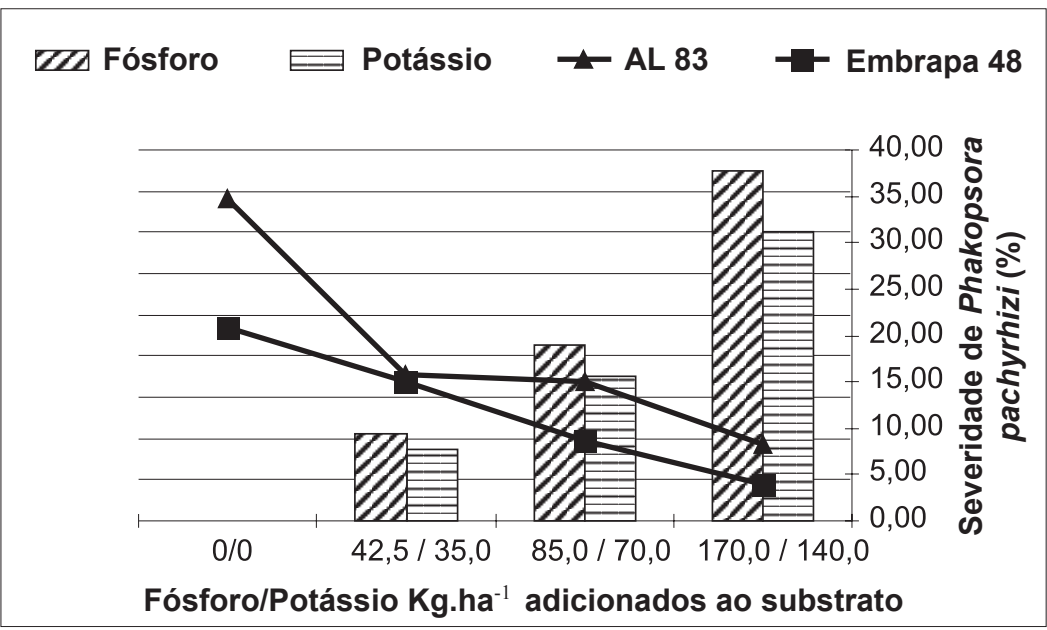

FIG. 1 - Influência dos diferentes níveis de adubação $\left[\mathrm{P}_{2} \mathrm{O}_{5}\left(8,4 \mathrm{mg} \mathrm{dm}{ }^{-3}\right)\right.$ e $\mathrm{K}_{2} \mathrm{O}(65$ $\left.\mathrm{mg} \mathrm{dm}^{-3}\right)$ ] sobre a expressão de resistência parcial das cultivares de soja Embrapa 48 e Al 83 no estádio fenológico V2.

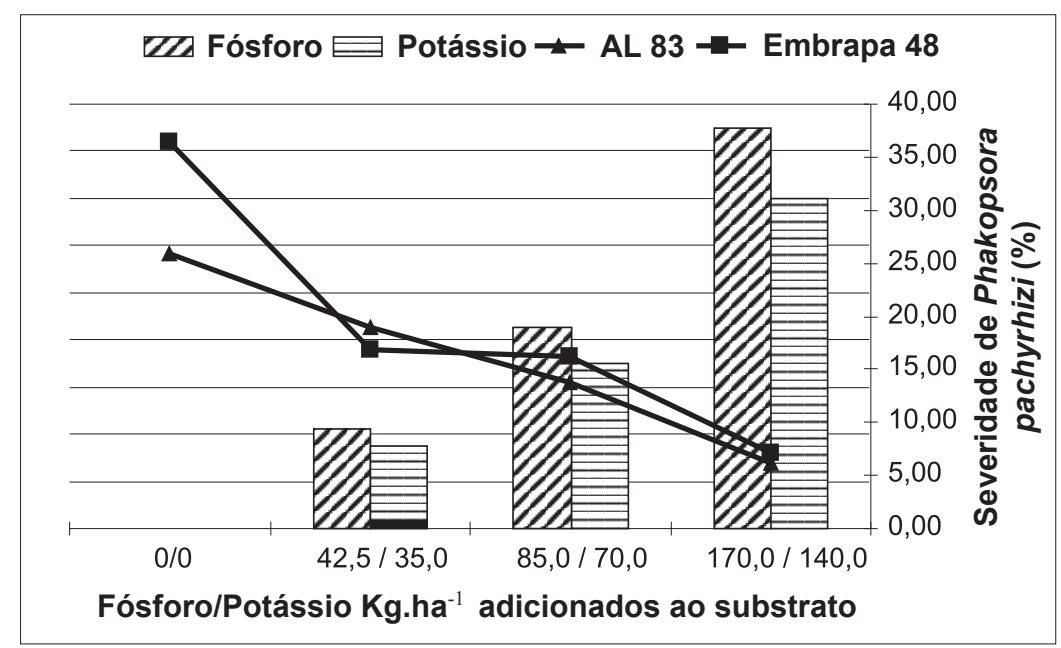

FIG. 2 - Influência dos diferentes níveis de adubação $\left[\mathrm{P}_{2} \mathrm{O}_{5}\left(8,4 \mathrm{mg} \mathrm{dm}^{-3}\right)\right.$ e $\mathrm{K}_{2} \mathrm{O}(65$ $\left.\mathrm{mg} \mathrm{dm}^{-3}\right)$ ] sobre a expressão de resistência parcial das cultivares de soja Embrapa 48 e Al 83 no estádio fenológico R5. 


\section{REFERÊNCIAS BIBLIOGRÁFICAS}

ANNONE, J.G., GARCIA, R. \& COPIA, P. Efecto sinérgico de fertilización nitrogenada y tratamiento fungicida sobre la reducción de síntomas de la mancha amarilla del trigo. XIII Congresso Latinoamericano de Fitopatologia. Córdoba, Argentina, 2005. p.246. (Resumo)

BLOOM, A.J. Nutrição Mineral. In: Taiz, L. \& Zeiger, E. (Eds.) Fisiologia Vegetal. $3^{\circ}$ ed, 2004. p. 96-103.

CAMPBELL, C.L. \& MADDEN, L.V. Crop loss assessment and modeling. In: Campbell, C.L. \& Madden, L.V. (Eds.) Introduction to plant disease epidemiology. New York NY. John Wiley \& Sons. 1990. p. 393-422.

DALLAGNOL, L.J., BALARDIN, R.S. \& UGALDE, M.G. Reação de cultivares de soja a ferrugem da soja (Phakopsora pachyrhizi). Anais, XIX Jornada Acadêmica Integrada, 2004, Santa Maria. RS, 2004. CD Rom.

DEVELASH, R.K. \& SUGHA, S.K. Factors affecting development of downy mildew (Peronospora destructor) of onion (Allium cepa). Indian Journal of Agricultural Sciences 67:71-74. 1997.

GARCIA JUNIOR, D., POZZA, E.A, POZZA, A.A.A., SOUZA, P.E., CARVALHO, J.G. \& BALIEIRO, A.C. Incidência e severidade da cercosporiose do cafeeiro em função do suprimento de potássio e cálcio em solução nutritiva. Fitopatologia Brasileira 28:286-291. 2003.

GUPTA, G.K. \& SINGH, D. Downy mildew control in pearl millet through nutrient application. Indian Phytopathology 48:439-443. 1995.

HARTMAN, G. L., WANG, T.C. \& TCHANZ, A. T. Soybean rust development and the quantitative relationship between rust severity and soybean yield. Plant Disease 75:596-600. 1991.

HUBER, D.M. Relationship between mineral nutrition of plants and disease incidence. In: Workshop - Relação entre nutrição de plantas e incidência de doenças. Piracicaba: Potafós, 08 e 09/05/2002, Anais e vídeo..., CD-ROM - vídeo 01, 2002.

ITO, M.F., MASCARENHS, H.A.A., TANAKA, M.A.S., DUDIENAS, C., TANAKA, R.T., GALLO, P.B. \& MIRANDA, M.A.C. Efeito residual da adubação potássica e da calagem sobre a incidência de Phomopsis spp. em sementes de soja. Fitopatologia Brasileira 19:44-49. 1994.
KIM, S.G., KIM, K.W., PARK, E.W. \& CHOI, D. Silicon-induced cell wall fortification of rice leaves: A possible cellular mechanism of enhanced host resistance to blast. Phytopathology 92:10951103. 2002.

LEVY, C. Zimbabwe - a country report on soybean rust control. In: World Soybean Research Conference, VII, Proceedings, 2002, Foz do Iguaçu, p.340-348.

COMISSÃO DE QUÍMICA E FERTILIDADE DO SOLO. Manual de adubação e calagem para os estados do Rio Grande do Sul e Santa Catarina / Sociedade Brasileira de Ciência do Solo. 10.ed. - Porto Alegre, 2004.

MARSCHNER, H. Relations hip between mineral nutrition and plant disease and pests. In: Marschner, H. (Ed.) Mineral nutrition of higher plants. London. Academic Press. 1996. p.369-390.

MASCARENHAS, H.A.A., MIRANDA, M.A.C., BATAGLIA, O.C. TISSELLI FILHO, O., BRAGA, N.R. \& SOAVE, J. Efeito da adubação potássica sobre o ataque da soja pelo Diaporthe phaseolorum (Cke. \& Ell.) Sacc. Var. sojae (Lehman)Wehm. Summa Phytopathologica 2:230-234. 1976.

MEURER, E.J. \& INDA Jr, A.V. Potássio e adubos potássicos. In: Bissani, C.A., Gianello, C., Tedesco, M.J. \& Camargo, F.A.O. (Eds.) Fertilidade dos solos e manejo da adubação de culturas. Porto Alegre RS. Gênesis, 2004. p.139 - 152.

NAVA-JUARES, R.A., DOMINGUES MUÑOZ, E., MONTESBELMONT, R. \& FLORES-MOCTEZUMA, H.E. Fuentes y dosis de nitrógeno en el control de Sclerotium rofsii en cebolla en Morelos, México. XIII Congresso Latinoamericano de Fitopatologia. Cordoba, Argentina, 2005. p.302. (Resumo)

POTAFOS - Associação Brasileira Para Pesquisa Da Potassa E Do Fosfato. Como a planta de soja se desenvolve. Arquivo do Agrônomo - $\mathrm{N}^{\circ}$ 11. 1997. 21p. Traduzido do original: How a soybean Plant Develops. Special Report no 53. Iowa. June, 1997.

POZZA, E.A. \& POZZA, A.A.A. Manejo de doenças de plantas com macro e micronutrientes. XXXVI Congresso Brasileiro de Fitopatologia, Manejo Integrado de Doenças de Plantas. Uberlândia, 2003. (Resumo)

REUVENI, M., NAOR, A., REUVENI, R., SHIMONI, M. \& BRAVDO, B. The influence of NPK fertilization rates on susceptibility to powdery mildew of field-grown wine grapes. Journal of Small Fruit and Viticulture 2:31-41. 1993. 